J. Clin. Chem. Clin. Biochem.

Vol. 20, 1982, pp. 515-519

\title{
Hochempfindlicher Enzymimmunoassay zur Bestimmung von Human-Pankreas-Lipase
}

Von G. Grenner, Gerlinde Deutsch, R. Schmidtberger und F. Dati

Forschungslaboratorien der Behringwerke AG, Marburg/Lahn

(Eingegangen am 5. Februar/21. April 1982)

Herrn Professor Weissermel zum 60. Geburtstag gewidmet

Zusammenfassung: Es wird ein hochempfindlicher und spezifischer Enzymimmunoassay zur Bestimmung der PankreasLipase in Seren und Plasmen beschrieben. Der Test ist nach dem Sandwich-Verfahren aufgebaut, die Gesamtinkubationszeit beträgt 4,5 Stunden bei $20-25^{\circ} \mathrm{C}$. Der Meßbereich liegt zwischen 3 und $300 \mu \mathrm{g} / 1 \mathrm{Lipase}(1+10$-Verdünnung der Proben), als untere Nachweisgrenze wurden 0,3 $\mu \mathrm{g} / 1$ ermittelt $(1+1$-Verdünnung der Probe). Für die Präzision in der Serie ergaben sich Variationskoeffizienten zwischen 2,9 und 6,5\%, von Tag zu Tag wurden Werte zwischen 4,4 und $10,5 \%$ gefunden.

Aus der Lipase Bestimmung in 369 Seren von gesunden Erwachsenen errechnete sich ein Referenzbereich von 7,7 bis $56 \mu \mathrm{g} / 1$ (2,5.-97,5. Perżentil, Median $23 \mu \mathrm{g} / \mathrm{l})$. Im Nabelschnurserum wurden sehr niedrige Konzentrationen bestimmt (Median 2,8 $\mu \mathrm{g} / 1$ ). Zwischen 3 und 50 Jahren bleibt die Lipasekonzentration annähernd konstant (Median 15-20 $\mu \mathrm{g} / 1$ ), bis zum 70. Lebensjahr erfolgt dann ein Anstieg des Median auf $26 \mu \mathrm{g} / 1$.

\section{A highly sensitive enzyme immunoassay for the determination of pancreatic lipase}

Summary: A highly sensitive and specific enzyme immunoassay for the determination of pancreatic lipase is described. The test follows the sandwich principle; total incubation time is 4.5 hours at $20-25^{\circ} \mathrm{C}$. Lipase concentrations between 3 and $300 \mu \mathrm{g} / 1$ can be quantified (sample dilution $1+10$ ); the lower detection limit is $0.3 \mu \mathrm{g} / 1$ (sample dilution $1+1$ ). Coefficients of variation from 2.9 to $6.5 \%$ for the in batch precision, and from 4.4 to $10.5 \%$ for the day-to day precision were found.

A reference range from 7.7 to $56 \mu \mathrm{g} / \mathrm{l}$ was calculated from the lipase concentrations in 369 serum samples from healthy adults $(2.5$ th -97.5 th percentile, median $23 \mu \mathrm{g} / 1)$. Very low concentrations were found in cord blood (median $2.8 \mu \mathrm{g} / \mathrm{l}$ ). The lipase level remains quite constant between the ages of 3 and 50 years (median $15-20 \mu \mathrm{g} / 1$ ); at the age of 70 years a median of $26 \mu \mathrm{g} / 1$ is reached.

\section{Einleitung}

Die menschliche Pankreas-Lipase (EC 3.1.1.3) ist ein Lipoprotein mit einem Molekulargewicht von 40000$46000(1,2,3)$, das Glycerinester langkettiger Fettsäuren hydrolisiert. Beim Übergang des Enzyms von der Bauchspeicheldrüse zum Gastrointestinaltrakt wird eine geringe Menge ins Blut abgegeben. Bei bestimmten Erkrankungen der Bauchspeicheldrüse, wie z.B. akuter Pankreatitis, wird eine erhöhte Konzentration von Lipase im Serum beobachtet.

Neben der Bestimmung der $\alpha$-Amylase, des immunreaktiven Trypsins und des Chymotrypsins im Stuhl hat die Lipase-Bestimmung in der Diagnostik und Verlaufskontrolle von Pankreaserkrankungen eine große Bedeutung. Pankreas-Lipase wird zur Zeit in den klinisch-che- mischen Labors mittels kommerzieller turbidimetrischer Verfahren (z.B. 4, 5) oder apparativ aufwendiger Titrationsmethoden $(6,7)$ bestimmt. Diese Methoden sind zum Teil nicht spezifisch für die Pankreas-Lipase, außerdem sind sie aufgrund ihrer relativ geringen Empfindlichkeit nur zur Messung erhöhter Lipasekonzentrationen einsetzbar. Messungen im Normalbereich können nur ungenau durchgeführt werden, bei subnormalen Konzentrationen versagen diese Methoden.

Eine immunchemische Methode zur Bestimmung der Lipase, wie z.B. ein Enzymimmunoassay, ist unabhängig von spezifischen Substraten, Cofaktoren und anderen Lipasen und sollte bei ausreichender Empfindlichkeit auch eine exakte Erfassung von Lipase im normalen und subnormalen Konzentrationsbereich erlauben. 


\section{Material und Methoden}

\section{Probenmaterial}

Serum- und Plasmaproben, die zur Ermittlung der Referenzwerte verwendet wurden, stammten von gesunden Blutspendern ohne klinische Symptome. Seren von Patienten mit PankreasErkrankungen und Serumproben von Gesunden vor und nach Heparingabe zur Induktion von post-Heparin-Lipasen wurden uns von verschiedenen Kliniken zur Verfügung gestellt.

\section{Reagenzien}

Anti-Lipase-Antiseren wurden durch Immunisierung von Schafen und Kaninchen mit hochgereinigter Lipase (2) (spezifische Aktivität $2000 \mathrm{IU} / \mathrm{mg}$, Triolein als Substrat bei $25^{\circ} \mathrm{C}$ ) gewonnen. Antikörper vom Schaf wurden zur Herstellung von Antikörper-beschichteten 2 ml-Kunststoffröhrchen verwendet (8), Antikörper vom Kaninchen wurden mit Peroxidase nach der Metaperiodat-Methode (9) konjugiert. Das Konjugat wurde in $50 \mathrm{mmol} / 1$ Tris-HCl-Puffer, der $100 \mathrm{ml} / 1$ Rinderserum enthielt, auf eine Konzentration von $0,5 \mathrm{mg} / 1$ verdünnt. Als Standards wurden Verdünnungen von reiner Lipase in einem Lipase-freien Humanserum bzw. in $50 \mathrm{mmol} / \mathrm{l}$ Tris-HCl-Puffer $\mathrm{pH} \mathrm{7,4,} \mathrm{der}$ $10 \mathrm{~g} / 1$ Humanalbumin enthielt, eingesetzt (Konzentration $0-300 \mu \mathrm{g} / 1$ ). Lipase-freies Serum wurde durch Behandlung von normalem Humanserum mit trägergebundenen Antikörpern gegen Lipase gewonnen.

Zur Vorverdünnung der Proben wurde Rinderserum, das $50 \mathrm{mmol} / 1$ Tris-HCl-Puffer $\mathrm{pH}$ 7,4 enthielt (Inkubationsmedium), verwendet. Die Waschlösung bestand aus Phosphat-gepufferter $\mathrm{NaCl}$-Lösung pH 7,2, die das Detergens Tween ${ }^{\circledR} 20$ enthielt. Die an der Röhrchenwand gebundene Peroxidase-Aktivität bestimmten wir mit $15 \mathrm{mmol} / 1 \mathrm{o}$-Phenylendiamin und $7 \mathrm{mmol} / 1$ Wasserstoffperoxid in $200 \mathrm{mmol} / 1$ Citrat-Phosphat-Puffer pH 5,0 (Chromogenlösung). Zum Unterbrechen der enzymatischen Katalyse wurde $250 \mathrm{mmol} / 1 \mathrm{Sch}$ wefelsäure verwendet.

\section{Testdurchführung}

Der Enzymimmunoassay zur Lipase-Bestimmung ist nach dem Sandwich-Prinzip aufgebaut. Während der ersten Inkubation wurde die Lipase an Antikörper, die an der Oberfläche der Kunststoffröhrchen fixiert sind, gebunden. In der zweiten Stufe reagierte der Peroxidase-konjugierte Antikörper gegen Lipase mit den noch freien Antigendeterminanten, anischließend wurde die gebundene Enzymaktivität bestimmt. Tabelle 1 zeigt die Testdurchfürung.

Die Standards wurden $1+10$, Serumproben entsprechend der zu erwartenden Lipase-Konzentration $1+1,1+10$ oder $1+100$ in Inkubationsmedium verdünnt. Die Lipasekonzentrationen im Serum wirden an der Referenzkurve abgelesen.

\section{Ergebnisse}

\section{Meßbereich und Nachweisgrenze}

Abbildung 1 zeigt eine typische Kalibrierkurve für den Enzymimmunoassay zur Bestimmung der PankreasLipase. Im Bereich zwischen 3 und $300 \mu \mathrm{g} / 1$ bei einer $1+10$-Verdünnung der Standards ergibt sich bei doppelt-logarithmischer Auftragung eine fast lineàre Beziehung zwischen Konżentration und Absorption. Die Standards werden generell $1+10$ verdünnt. Bei $1+1$ Verdünnung der Proben ergibt sich ein Meßbereich von 0,6-60 $\mu \mathrm{g} / 1$, bei $1+10$-Verdünnung von $3-300 \mu \mathrm{g} / 1$ und bei einer $1+100-V e r d u ̈ n n u n g$ von $30-3000 \mu \mathrm{g} / 1$. Da sich keine Unterschiede in der Signalhöhe bei Verwendung von Standards auf Serum- oder Albumin-

Tab. 1. Testdurchfuhrung des Enzymimmunoassays Lipase.

\begin{tabular}{|c|c|c|c|c|c|c|c|c|c|c|c|c|c|c|c|c|}
\hline \multirow[b]{2}{*}{ Ansatz Nr.: } & \multicolumn{10}{|c|}{ Standardproben } & \multicolumn{2}{|c|}{$\begin{array}{l}\text { Kontroll- } \\
\text { proben }\end{array}$} & \multicolumn{4}{|c|}{$\begin{array}{l}\text { Patienten- } \\
\text { proben }\end{array}$} \\
\hline & 1 & 2 & 3 & 4 & 5 & 6 & 7 & 8 & 9 & 10 & 11 & 12 & 13 & 14 & 15 & 16 etc. \\
\hline $\begin{array}{ll}\mu \mathrm{l}: & \\
\text { Standard*) } & S_{1} \\
\text { Standard } & S_{2} \\
\text { Standard } & S_{3} \\
\text { Standard } & S_{4} \\
\text { Standard } & S_{5} \\
\text { Kontrollserum } \\
\text { Patientenseren }\end{array}$ & 200 & 200 & 200 & 200 & 200 & 200 & 200 & 200 & 200 & 200 & 200 & 200 & 200 & 200 & 200 & 200 \\
\hline
\end{tabular}

Inkubation 2 Stunden bei $20-25^{\circ} \mathrm{C}$ in Antikörper-beschichteten Kunststoffröhrchen Absaugen und einmal waschen

Anti-Lipase / Peroxidase-Konjugat ( $\mu$ l)

Inkubation 2 Stunden bei $20-25^{\circ} \mathrm{C}$

Absaugen und $\mathrm{zweimal}$ waschen

Chromogen-Substrat-Lösung ( $\mu \mathrm{l})$

Inkubation 30 Minuten bei $20-25^{\circ} \mathrm{C}$

Stopplösung $(\mu \mathrm{l})$

Messung der Absorption bei $492 \mathrm{~nm}$

*) Standard-Konzentrationen: $\begin{array}{lll}S_{1} 3 \mu \mathrm{g} / 1 & S_{4} 100 \mu \mathrm{g} / 1 \\ & S_{2} 10 \mu \mathrm{g} / 1 & S_{5} 300 \mu \mathrm{g} / 1 \\ & S_{3} 30 \mu \mathrm{g} / 1\end{array}$


Matrix zeigten, wurden für alle weiteren Untersuchunger. Standards auf Albuminbasis verwendet.

Von 60 untersuchten pathologischen Seren mit erhöhter Lipasekonzentration lagen $64 \%$ unter $300 \mu \mathrm{g} / 1,33 \% \mathrm{im}$ Bereich zwischen 300 und $3000 \mu \mathrm{g} / 1$ und $3 \%$ über $3000 \mu \mathrm{g} / 1$. Der höchste gemessene Wert lag bei $5000 \mu \mathrm{g} / 1$.

Die untere Nachweisgrenze, ausgedrückt als das Dreifache der Standardabweichung bei der Lipasekonzentration Null (Lipase-freies Serum) beträgt etwa $0,3 \mu \mathrm{g} / 1$ bei einer $1+1$-Verdünnung der Probe bzw. etwa $1,5 \mu \mathrm{g} / 1$ bei einer $1+10$-Verdünnung.

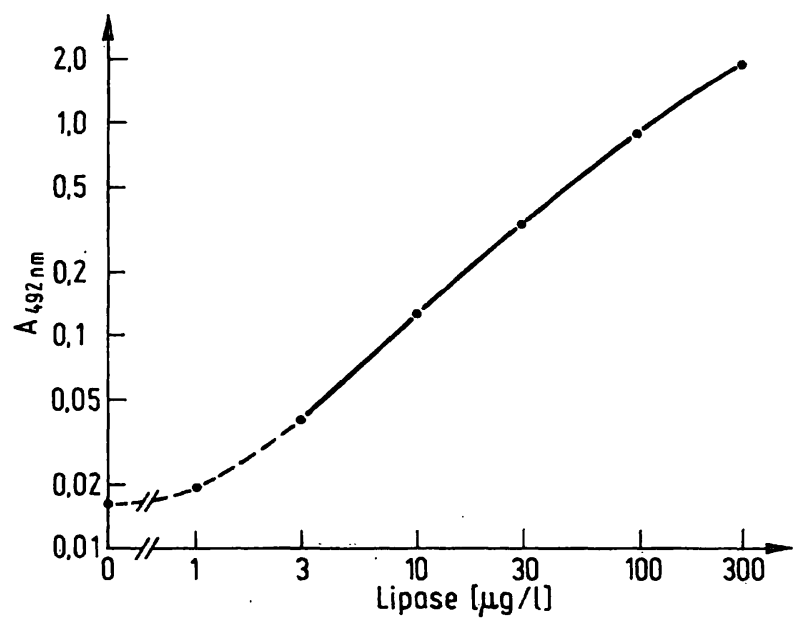

Abb. 1. Kalibrierkurve Enzymimmunoassay Lipase.

\section{Präzision}

Die Ergebnisse zur Präzision des Tests sind in Tabelle 2 zusammengestellt. Sie wurden an Seren mit Lipase-Konzentrationen zwischen 7,5 und $250 \mu \mathrm{g} / 1$ ermittelt. In der Serie ergaben sich Vàriationskoeffizienten zwischen 2,9 und $6,5 \%$, von Tag żu Tag wurden Werte zwischen 4,4 und $10.5 \%$ erhalten.

Tab. 2. Reproduzierbarkeit dẹ Enzymimmunoassays L̇ipase.

\begin{tabular}{ll}
\hline Präzision in der Serie $(n=20$ Einzelbestimmungen): \\
\hline Lipase-Kọnentration & Variationskoeffizient \\
$(\mu g / 1)$ & $(\%)$ \\
7,5 & 5,3 \\
24 & 3,5 \\
92 & 2,9 \\
247 & 6,5 \\
\hline
\end{tabular}

Präzision von Tag zu Tag $(\mathrm{n}=10$ Tage)*):

\begin{tabular}{ll}
\hline $\begin{array}{l}\text { Lipase-Konzentration } \\
(\mu \mathrm{g} / 1)\end{array}$ & Variationskoeffizient \\
& $(\%)$ \\
8,0 & \\
26 & 9,5 \\
87 & 7,3 \\
25.5 & 4,4 \\
\hline
\end{tabular}

*) Jeweils 1 Doppelbestimmung an 10 Tagen

\section{Wiederfindung, Linearität}

Die Wiederfindungsraten bei Aufstockversuchen von verschiedenen Humanseren mit Pankreas-Lipase sind in Tabelle 3 zusammengestellt, sie liegen zwischen 92 und $106 \%$.

Tab. 3. Wiederfindung im Enzymimmunoassay Lipase.

\begin{tabular}{|c|c|c|c|c|c|c|c|}
\hline \multirow{3}{*}{$\begin{array}{l}\text { Pro- } \\
\text { be } \\
\text { Se- } \\
\text { rum } \\
\text { Nr. }\end{array}$} & \multirow{3}{*}{$\begin{array}{l}\text { Lipase- } \\
\text { Kon- } \\
\text { zentra- } \\
\text { tion } \\
(\mu \mathrm{g} / 1)\end{array}$} & \multicolumn{6}{|c|}{ Wiederfindung bei Zusatz von } \\
\hline & & \multicolumn{3}{|c|}{$25 \mu \mathrm{g} / 1$} & \multicolumn{3}{|c|}{$100 \mu \mathrm{g} / 1$} \\
\hline & & $(\mu \mathrm{g} / 1)$ & $(\mu \mathrm{g} / 1)$ & $\begin{array}{l}\text { Anteil } \\
\text { des } \\
\text { Soll- } \\
\text { wertes }\end{array}$ & $(\mu \mathrm{g} / 1)$ & $(\mu \mathrm{g} / 1)$ & $\begin{array}{l}\text { Anteil } \\
\text { des } \\
\text { Soll- } \\
\text { wertes }\end{array}$ \\
\hline $\begin{array}{l}1 \text { (Li- } \\
\text { pase- } \\
\text { frei) }\end{array}$ & 0 & 24,5 & 25 & 0,98 & 102 & 100 & 1,02 \\
\hline $\begin{array}{l}2 \\
3 \\
4 \\
5\end{array}$ & $\begin{array}{c}19,5 \\
40 \\
95 \\
152\end{array}$ & $\begin{array}{c}46,5 \\
69 \\
114 \\
163\end{array}$ & $\begin{array}{l}44,5 \\
65 \\
120 \\
177\end{array}$ & $\begin{array}{l}1,04 \\
1,06 \\
0,95 \\
0,92\end{array}$ & $\begin{array}{l}115 \\
147 \\
191 \\
235\end{array}$ & $\begin{array}{l}119,5 \\
140 \\
195 \\
252\end{array}$ & $\begin{array}{l}0,96 \\
1,05 \\
0,98 \\
0,93\end{array}$ \\
\hline
\end{tabular}

Angegebene Lipase-Konzentrationen sind Mittelwerte aus Doppelbestimmungen in drei Serien.

Zur Prüfung auf Linearität bei Verdünnung (Verdünnungsechtheit) wurden Seren mit Inkubationsmedium in verschiedenen Verhältnissen verdünnt und die Lipase-Konzentration im Enzymimmunoassay bestimmt. Aus Tabelle 4 ist zu entnehmen, daß keine Beeinflussung des Ergebnisses durch unterschiedliche Verdünnungsverhältnisse eintritt.

Tab. 4. Linearität des Enzymimmunoassays Lipase.

\begin{tabular}{llll}
\hline Probe & Probenverdünnung & $\begin{array}{l}\text { Kon- } \\
\text { zentration } \\
\text { im Testansatz } \\
(\mu \mathrm{g} / \mathrm{l})\end{array}$ & $\begin{array}{l}\text { Kon- } \\
\text { zentration } \\
\text { in der Probe } \\
(\mu \mathrm{g} / \mathrm{l})\end{array}$ \\
\hline A & & 14,5 & 29 \\
& $1: 2$ & 2,7 & 27 \\
B & $1: 10$ & 12,2 & 24,5 \\
& $1: 2$ & 2,5 & 25 \\
C & $1: 10$ & 4,1 & 20,5 \\
& $1: 5$ & 2,25 & 22,5 \\
& $1: 10$ & 0,41 & 20,5 \\
D & $1: 50$ & 24 & 120 \\
& $1: 5$ & 11,5 & 115 \\
& $1: 10$ & 2,3 & 115 \\
E & $1: 50$ & 27 & 1350 \\
& $1: 50$ & 14,5 & 1450 \\
\hline
\end{tabular}

\section{Verwendbarkeit von Plasmen}

Von gesunden Spendern wurden am gleichen Tag Serum, Heparin-, EDTA- und Citratplasma abgenommen. Es konnte keine Beeinflussung der enzymimmunologischen Lipase-Bestimmung durch Antikoagulantien festgestellt werden. 


\section{Spezifität}

Nach intravenöser Heparingabe werden im menschlichen Serum erhöhte Konzentrationen an LipoproteinLipase und hepatischer Triglycerid-Lipase gemessen. In Tabelle 5 sind die Ergebnisse der Untersuchung von Proben vor und nach Induktion zusammengestellt. Die Heparin-induzierten Lipasen wurden radioimmunologisch (10), die Pankreas-Lipase mit dem beschriebenen Enzymimmunoassay gemessen. Die Konzentration der Pankreas-Lipase änderte sich nach Heparingabe nur geringfügig.

Tab. 5. Spezifität des Enzymimmunoassays zur Pankreas-LipaseBestimmung.

\begin{tabular}{|c|c|c|c|c|c|c|}
\hline \multirow{3}{*}{$\begin{array}{l}\text { Probe } \\
\text { Nr. }\end{array}$} & \multicolumn{6}{|c|}{ Konzentration } \\
\hline & \multicolumn{2}{|c|}{$\begin{array}{l}\text { Lipoprotein- } \\
\text { Lipase } \\
(\mathrm{mg} / \mathrm{l})\end{array}$} & \multicolumn{2}{|c|}{$\begin{array}{l}\text { Hepatische } \\
\text { Triglycerid-Lipase } \\
(\mathrm{mg} / \mathrm{l})\end{array}$} & \multicolumn{2}{|c|}{$\begin{array}{l}\text { Pankreas- } \\
\text { Lipase } \\
(\mu \mathrm{g} / 1)\end{array}$} \\
\hline & $\begin{array}{l}\text { vor } \\
\text { Hepa }\end{array}$ & $\begin{array}{l}\text { nach } \\
\text { ngabe }\end{array}$ & $\begin{array}{l}\text { vor } \\
\text { Hepa }\end{array}$ & $\begin{array}{l}\text { nach } \\
\text { igabe }\end{array}$ & $\begin{array}{l}\text { vor } \\
\text { Hep }\end{array}$ & $\begin{array}{l}\text { nach } \\
\text { ngabe }\end{array}$ \\
\hline $\begin{array}{l}1 \\
2 \\
3 \\
4\end{array}$ & $\begin{array}{l}0,09 \\
0,32 \\
0,20 \\
0,37\end{array}$ & $\begin{array}{l}5,9 \\
6,1 \\
3,8 \\
4,4\end{array}$ & $\begin{array}{l}0,28 \\
- \\
\overline{0} \\
0,65\end{array}$ & $\begin{array}{l}23,2 \\
18,9 \\
17,1 \\
27,2\end{array}$ & $\begin{array}{l}21 \\
25 \\
7,8 \\
15\end{array}$ & $\begin{array}{l}25 \\
28 \\
8,8 \\
17\end{array}$ \\
\hline
\end{tabular}

\section{Stabilität der Antigenität von Pankreas-Lipase im Serum}

Humanseren wurden bei $+4{ }^{\circ} \mathrm{C}$ und bei $+25^{\circ} \mathrm{C}$ über 4 Wochen gelagert. Die Lipase-Konzentrationen wurden im Enzymimmunoassay bestimmt. Im Vergleich zu bei $-70^{\circ} \mathrm{C}$ gelagerten Proben dieser Seren ergab sich kein Konzentrationsabfall. Auch mehrfaches Einfrieren (5fach) hatte keinen Einfluß auf die ermittelten LipaseKonzentrationen.

\section{Referenzbereich, Altersabhängigkeit der Lipase- Konzentration}

In 369 Seren von gesunden Blutspendern (Alter 1865 Jahre, 205 männlich, 164 weiblich) wurde der Lipasegehalt bestimmt. $95 \%$ aller Werte $(2,5$. bis 97,5 . Perzentil) liegen im Bereich zwischen 7,7 und $56 \mu \mathrm{g} / \mathrm{l}$. Als Median (50. Perzentil) wurde ein Wert von $23 \mu \mathrm{g} / \mathrm{l}$ ermittelt.

Weißhaar et al. (11) fanden, daß die Lipase-Konzentration mit zunehmendem Alter ansteigt. Die Bestimmung erfolgte mittels Turbidimetrie. Angaben über Konzentrationen bei Neugeborenen und im Kindesalter liegen nicht vor.

Zur Feststellung einer Altersabhängigkeit wurde die Lipase-Konzentration in Nabelschnurseren und in Seren von Kindern und Erwachsenen mit Hilfe des Enzymimmunoassays bestimmt. 150 Nabelschnurseren, jeweils 20 Seren je Lebensjahr im Bereich von 1 bis 10 Jahren und 20 Seren für jedes Lebensjahrzehnt im Bereich von 11 bis 70 Jahren (11-20, 21-30 usw. bis 6170 Jahre) wurden untersucht. Die Ergebnisse sind in
Abbildung 2 dargestellt (doppelt-logarithmische Auftragung). Bei Neugeborenen ist im Nabelschnurserum nur eine sehr geringe Konzentration bestimmbar (Median 2,8 $\mu \mathrm{g} / \mathrm{l}$ ). Bis zum 3. Lebensjahr steigt der Lipasespiegel im Serum an, der Median liegt bei $15 \mu \mathrm{g} / \mathrm{l}$. Zwischen dem 3. und 50. Lebensjahr bleibt die LipaseKonzentration annähernd konstant. Im Lebensabschnitt zwischen 51 und 70 Jahren tritt eine stärkere Erhöhung ein (Median $26 \mu \mathrm{g} / \mathrm{l}$ ). Der Streubereich der gemessenen Lipase-Konzentration läßt sich an den in Abbildung 2 eingezeichneten 5. und 95. Perzentilen erkennen.

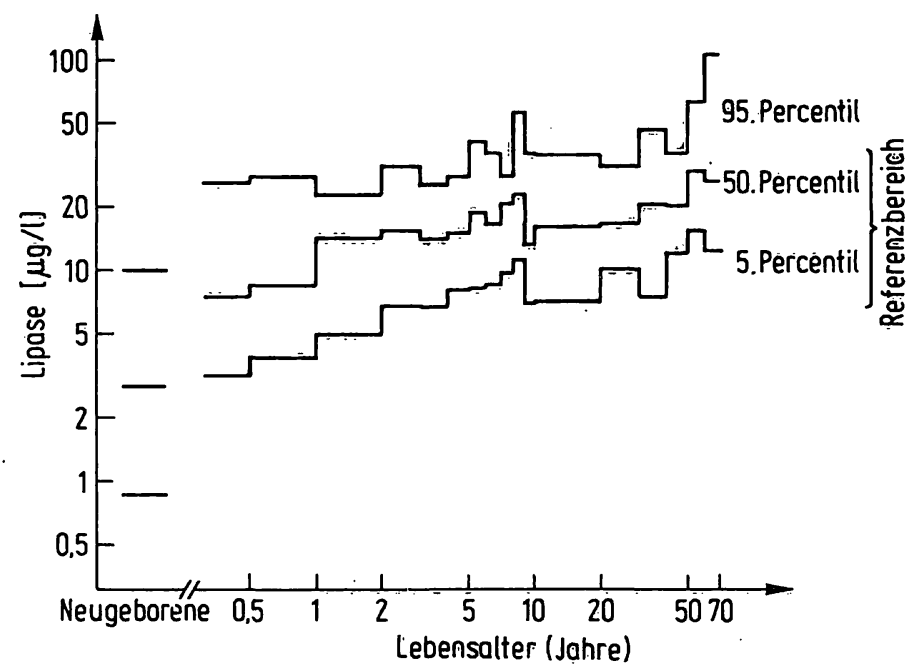

Abb. 2. Altersabhängigkeit der enzymimmunologisch bestimmten Lipase-Konzentration im Serum.

\section{Diskussion}

Zur Bestimmung der Mehrzahl der in der klinisch-chemischen Diagnostik wichtigen Enzyme ist die Messung der katalytischen Aktivität die Methode der Wahl. Solche Tests sind schnell und einfach durchführbar und lassen sich leicht automatisieren. Die Grenzen dieser Verfahren treten bei der Bestimmung von Isoenzymen und bei Enzymen mit geringer katalytischer Aktivität zutage. Auch das Fehlen photometrisch gut erfaßbarer Substrate erschwert gelegentlich die Entwicklung einfacher Tests.

Versuche, zur Lipase-Bestimmung unphysiologische Substrate einzusetzen $(12,13)$, führten zu unspezifischen Tests, da auch andere Hydrolasen angezeigt wurden. Ein Trübungstest (14) ist recht spezifisch, ca.. 4\% der Seren zeigen jedoch eine inverse Reaktionskinetik, solche Proben sind dann nicht bestimmbar. Diese Methoden sind ebenso wie die Titrationsmethoden $(6,7)$ nicht empfindlich genug, um im Normalbereich und insbesondere bei sehr niedrigen Lipase-Konzentrationen präzise Aussagen zu liefern.

Ähnlich wie bei der immunchemischen Bestimmung der sauren Prostata-Phosphatase (15) sollte ein Immunoassay die Pankreas-Lipase-Bestimmung empfindlicher und spezifischer machen.
$-8$ 
Bislang wurden nur Immunpräzipitationsmethoden zur Pankreas-Lipase-Bestimmung beschrieben (2), deren Empfindlichkeit jedoch zur Erfassung der niedrigen Konzentrationen im Serum nicht ausreicht.

Unsere positiven Erfahrungen mit den nach dem Sandwich-Verfahren aufgebauten Enzymimmunoassays zur Bestimmung von in sehr geringen Konzentrationen in Serum vorliegenden Proteinen wie z. B. Immunglobulin E (16), saure Prostata-Phosphatase (17) und $\alpha$-Foetoprotein (18) legten nahe, dieses Verfahren auch für die Bestimmung der Pankreas-Lipase anzuwenden. Mit einer Nachweisgrenze von $0,3 \mu \mathrm{g} / 1$ ist der Test so empfindlich, daß Konzentrationen unterhalb des Normalbereiches $(<7 \mu \mathrm{g} / \mathrm{l})$ ohne Probleme erfaßt werden können. Der Meßbereich erstreckt sich über zwei Dekaden, bei einer $1+10$-Vorverdünnung des Patientenserums von 3 bis $300 \mu \mathrm{g} / 1$. Patientenseren im niedrigeren $(<3 \mu \mathrm{g} / \mathrm{l})$ oder im höheren Konzentrationsbereich $(>300 \mu \mathrm{g} / 1)$ können erfaßt werden, wenn eine entsprechende Verdünnung $(1+1$ bzw. $1+100)$ gewählt wird. Voraussetzung für ein solches Verfahren ist die hohe Wiederfindungsrate (Tab. 3) und die gute Linearität des Testes (Tab. 4).

Lipasen nicht-pankreatischen Ursprungs, wie z.B. die Lipoprotein-Lipase und hepatische Triglycerid-Lipase, werden vom Enzymimmunoassay nicht erfaßt. Ebenso werden keine Störungen bei hämolytischen, lipämischen und ikterischen Seren und hohen Rheumafaktor-Konzentrationen beobachtet (unveröffentlichte Ergebnisse).

Bei der Untersuchung der Altersabhängigkeit der LipaseKonzentration im Serum bestimmten wir sehr geringe Konzentrationen im Neugeborenenserum. Ein anderes
Pankreas-Enzym, das Trypsin, liegt auch schon bei Neugeborenen in ähnlich hoher Konzentration wie bei Erwachsenen vor (19).

Vom 3. bis zum 50. Lebensjahr bleibt nach unseren Untersuchungen die Lipase-Konzentration annähernd konstant, so daß der an einem Kollektiv von 369 Personen ermittelte vorläufige Referenzbereich von 7,7-56 $\mu \mathrm{g} / \mathrm{l}$ als weitgehend altersunabhängig angesehen werden kann.

Der beschriebene heterogene Enzymimmunoassay erlaubt eine spezifische, empfindliche und präzise $\mathrm{Be}$ stimmung der Pankreas-Lipase im Serum und Plasma.

Erste Untersuchungen an Seren von Pankreatitis-Patienten zeigten erwartungsgemäß erhöhte Lipase-Konzentrationen bei akuter Pankreatitis, während bei chronischem Krankheitsbild subnormale Werte gefunden wurden. Uber diese Ergebriisse soll an anderer Stelle ausfuhrlich berichtet werden. Weitere interessante Fragestellungen, wie z.B. die Aussagekraft des Tests bei Verlaufskontrollen, bei Pankreas-Karzinom und bei cystischer Fibrose werden zur Zeit bearbeitet.

\section{Danksagung}

Wir danken Herrn Dr. K. P. Ringel, Sektion für Angewandte Immunologie, Universität Kiel, und Herrn Dr. O. Schrecker, Institut für Herzinfarktforschung, Universität Heidelberg, für die Bereitstellung der Serumproben.

Herr Dr. Schrecker bestimmte freundlicherweise auch die Konzentrationen der Lipoprotein-Lipase und hepatischen TriglyceridLipase im Radioimmunoassay.

Für die Durchführung der experimentellen Arbeiten danken wir Frau $H$. Burk und Herrn $K$. Krauße.

\section{Literatur}

1. Straube, E., Schütt, Chr., Br̀ock, J. \& Mücke, D. (1974) Acta Biol. Med. Germ. 33, $263-274$.

2. Forssell, E. (1974) Ann. Acad. Sci. Fenn. Ser., V. Med. $166,1-36$.

3. Vandermeers, A., Vandermeers-Piret, M. C. \& Rathe, J. (197.4) Biochim. Biophys. Acta 370, 257-268.

4. Ziegenhorn, J., Neumann, U. \& Knitsch, K. W. (1979) Clin. Chem. 25, 1067.

5. Temme, H. \& Róka, L. (1979) J. Clin. Chem. Clin. Biochem. 17, 193-194.

6. Rick, W. (1969) Z. Klin. Chem. Klin. Biochem. 7, 530-539.

7. Tietz, N. W. \& Fiereck, E. A. (1972) in Standard Methods of Clinical Chemistry (Cooper, G. R. ed.), New York Acad. Press, Vol. 7, pp. 19-31.

8. Catt, K. \& Tregear, G. W. (1967) Science 158, 1570-1572.

9. Nakane, P. K. \& Kawaoi, A. (1974) J. Histöchem. Cytochem. 22, 1084-1091.

10. Greten, H., De Grella, R., Klose, G., Rascher, W., de Gennes, J. L. \& Gjone, E. (1976) J. Lipid Research 17, 203-210.

11. Weißhaar, H. D., Sudhoff, H., Koller, P. U. \& Bablok, W. (1981) Dtsch. Med. Wochenschr. 106, 239-241.

12. Myrick, J. E. (1976) Dissertation, University of Alabama.

13. Fleisher, M. \& Schwartz, M. K. (1971) Clin. Chem. 17, 417.

14. Lorentz, K. \& Flatter, B. (1980) Med. Labor 33, 236-239.

15. Cooper, E. H., Glashan, R., Robinson, M. R. G. \& Trautner, K. (1981) Clin. Chim. Acta 113, 27-34.

16. Fateh-Moghadam, A., Neumeier, O., Von Stetten, K., Dati, F. \& Grenner, G. (1980) Z. Anal. Chem. 30, 123-124.

17. Grenner, G. (1980) Clin. Chem. 26, 987.

18. Dati, F., Grenner, G., Schmidtberger, R. (1981) J. Clin. Chem. Clin. Biochem. 19, 216.

19. Borgström, A., Sveger, T., Lindberg, T., Kullander, S. \& Svanberg, L. (1981) Acta Paediatr. Scand. 70, 619-621.

Dr. G. Grenner

Behringwerke AG

Postfach 1140

D-3550 Marburg 1 
。 